\title{
Soft X-ray imaging of spin dynamics at high spatial and temporal resolution
}

\author{
Brooke L. Mesler, Peter Fischer ${ }^{\mathrm{a}}$, Weilun Chao, and Erik H. Anderson \\ Center for X-ray Optics, Lawrence Berkeley National Laboratory, Berkeley, CA USA \\ Dong-Hyun Kim \\ Dept. Physics, Chungbuk National University, Cheongju 361-763, Korea
}

${ }^{\mathrm{a}}$ Corresponding author.

Tel.: + 1 510- 486 7052; fax: + 1510486 4550. E-mail address: PJFischer@lbl.gov (P. Fischer). 


\begin{abstract}
Soft X-ray microscopy provides element specific magnetic imaging with a spatial resolution down to $15 \mathrm{~nm}$. At XM-1, the full-field soft X-ray microscope at the Advanced Light Source in Berkeley, a stroboscopic pump and probe setup has been developed to study fast magnetization dynamics in ferromagnetic elements with a time resolution of 70 ps which is set by the width of the X-ray pulses from the synchrotron. Results obtained with a $2 \mu \mathrm{m} \times 4 \mu \mathrm{m} \times 45 \mathrm{~nm}$ rectangular permalloy sample exhibiting a seven domain Landau pattern reveal dynamics up to several nsec after the exciting magnetic field pulse. Domain wall motion, a gyrotropic vortex motion, and a coupling between vortices in the rectangular geometry are observed.
\end{abstract}

PACS: 07.85.Tt; 75.60.Ch; 75.40.Gb 


\section{Introduction}

Magnetism on the nanoscale is currently attracting significant fundamental interest due to proximity and confinement effects occurring at length scales which are comparable to material specific magnetic exchange lengths[1,2]. These have typical values on a sub$10 \mathrm{~nm}$ scale for commonly used elements, such as permalloy $\left(\mathrm{Fe}_{20} \mathrm{Ni}_{80}\right)$. This length scale is also of increasing technological interest in view of current achievements in ultrahigh density magnetic storage media and nanoscale magnetic sensor devices. To mention one example, the interlayer exchange coupling in ferromagnetic thin film structures giving rise to giant magnetoresistance effects (GMR) [3, 4] has found immediate application as read-heads in magnetic storage. While the GMR effect can be considered as a spin dependent magneto-transport, i.e. the magnetization of the sample impacts the propagation of the electric current in low dimensional magnetic system, the reverse effect, i.e. the spin torque produced by a spin polarized current acting on the magnetization in nanoscale elements $[5,6]$ is now considered as a new concept to manipulate magnetization in nanomagnetism. This might also have a huge potential in upcoming spintronic devices [7- 9]. One example here is the magnetic racetrack memory, which combines the advantages of magnetic and solid state memory in a 3 dimensional architecture [10].

Advanced analytical tools are urgently needed to enable both a fundamental understanding of the physical phenomena as well as to characterize technological devices and magnetic microscopies providing real space images with spatial resolution in the sub$10 \mathrm{~nm}$ regime are very attractive. 
In addition to the static magnetic properties on the nanoscale, fast dynamics extending deep into the fsec time scale, which is associated with magnetic exchange energies and can therefore be seen as a fundamental time scale, are of utmost interest. Technologically relevant time scales, such as the time to reverse magnetization in magnetic storage media, or the speed of domain walls in spin torque devices are in the nsec to psec time regime. Optical magnetic microscopies, such as time resolved Kerr microscopy using ultrafast laser sources are able to address the fsec or even asec time scales; however, their spatial resolution which is diffraction limited is in principle not sufficient for the nanoscale. Likewise, techniques such as Spin polarized Scanning Tunneling Microsopy which provide a nearly atomic spatial resolution do not offer sufficient time resolution. Soft X-ray microscopy using X-rays with wavelengths of a few nanometers produced at current high brightness synchrotron sources are able to combine both high spatial resolution determined by Fresnel zone plates used as X-ray optical elements and a fast time resolution limited only by the X-ray sources which have an inherent time structure in the 70ps regime [11].

This article describes the use of full field soft X-ray microscopy to study magnetization dynamics such as domain wall and vortex dynamics in rectangular micron sized $\mathrm{Fe}_{20} \mathrm{Ni}_{80}$ elements. It further details the experimental setup for time resolved imaging, which was developed recently at the instrument XM-1 at the Advanced Light Source (ALS) in Berkeley. Current synchrotrons do not provide sufficient photon flux per X-ray pulse to allow for single shot imaging; therefore, a stroboscopic pump-probe scheme is engaged where the time evolution of the spin dynamics is studied by varying the delay time 
between the magnetic excitation pump pulse and the X-ray probe pulse. This pumpprobe cycle is repeated at a $3 \mathrm{MHz}$ frequency for several minutes to collect one image.

\section{Experiment}

XM-1 is located at beamline 6.1.2 of the Advanced Light Source in Berkeley CA and uses radiation from a bending magnet with a useable energy range between 300 to $1300 \mathrm{eV}$ (Fig. 1). The microscope is capable of providing high spatial and temporal resolution as well as element specific magnetic contrast. At XM-1, X-rays are focused onto a sample and the transmitted X-rays are imaged at about 2000X magnification on a CCD camera.

Using apertures, an off-axis portion of the radiation beam can be selected, supplying elliptically polarized X-rays. This is essential for magnetic imaging, since the transmission of X-rays of a particular wavelength through a ferromagnetic sample is largely dependent on the relative orientation of the sample's magnetization and the polarization of the X-rays. This effect is known as X-ray Magnetic Circular Dichroism (XMCD) [14]. It occurs in the vicinity of material specific X-ray absorption edges and hence provides an (inherently) element specific magnetic contrast mechanism up to $25 \%$. The size of the XMCD effect, i.e. the magnetic contrast, exceeds its optical counterpart, i.e. the magneto-optical Kerr effect, by far, which in general is in the permille regime only. Since the XMCD effect depends on the projection of the magnetization vector onto the direction of the photon's helicity soft X-ray transmission microscopy can distinguish samples with perpendicular or in-plane magnetization by orienting the sample such that a component of the magnetization vector points along the axis of the incident beam. In 
addition, because $\mathrm{X}$-ray microscopy is a photon in, photon out technique, magnetic fields can be applied to the sample during the recording without disturbing the imaging properties of the microscope. Thus, e.g. images of magnetic structures in full hysteresis cycles can be recorded and features such as stochastic character of nucleation processes, switching field distributions of large arrays in patterned media, or the return-pointmemory effects can be studied with high spatial resolution.

The optical setup of a soft X-ray microscope is similar to an optical microscope; however, Fresnel zone plates (FZPs) are required and are the key components to enable imaging with soft X-rays. XM-1 consists of two Fresnel zone plate optics. The condenser zone plate (KZP) provides a hollow cone illumination of the sample and the micro zone plate (MZP) as a high resolution objective lens images the transmitted X-rays onto a 2048 x 2048 backside illuminated CCD camera. Because FZPs focus X-rays of different energies to different points, the KZP also serves, in tandem with a pinhole placed immediately before the sample, as a linear monochromator. The spectral resolution $\mathrm{E} / \Delta \mathrm{E}$ yields values of about 500, which is sufficient to separate the spin-orbit coupled $\mathrm{L}$ absorption edges in $3 \mathrm{~d}$ elements. The MZP is the objective lens of the microscope and provides the high spatial resolution. The outermost zone width of the MZP defines largely the resolution of XM-1. So far, at XM-1 FZPs with an outermost zone as small as $15 \mathrm{~nm}$ have been achieved $[12,13]$.

The time resolved experiments at XM-1 are performed during the 2-bunch mode operation of the ALS (Fig. 2). In this mode two electron bunches with a width of 70ps and separated by $328 \mathrm{~ns}$ distance from each other are circulating in the storage ring with a total electron current of about $40 \mathrm{~mA}$. This provides X-ray flashes at a frequency of 
$3 \mathrm{MHz}$ which constitute the probing pulse and also defines both the experimental clock and the time resolution in the experiment.

For a single X-ray image about $10^{10-11}$ photons are needed, taking into account about 1000 photons per CCD pixel and a typical $10 \%$ efficiency for each of the X-ray optics. Since at current synchrotrons the number of photons in a single bunch does not provide such photon flux, a stroboscopic scheme is required and the dynamic process of interest has to repeat perfectly down to a length scale corresponding to the spatial resolution of the X-ray microscope.

The evolution of the dynamics is then studied by varying the delay time between the excitation pump pulse and the X-ray probing pulse (Fig. 2).

The schematic diagram of the stroboscopic pump-probe scheme used for the time resolved experiment described here is shown in Fig. 3.

The local magnetization is excited by a fast magnetic field pulse triggered by the clock signal of the synchrotron which is provided in ECL logic. Therefore, the ferromagnetic sample is prepared onto a 100nm thin Au coplanar waveguide (Fig. 4). A fast electronic pulse with a rise time of about $100 \mathrm{ps}$ is generated by a pulser at $3 \mathrm{MHz}$ and launched into the waveguide structure to generate an Oersted field pulse. The output signal of the pulser is monitored by a fast oscilloscope which is triggered by the same clock signal as the pulser. A digital delay line allows the pump signal to be delayed up to a full $328 \mathrm{~ns}$ in $2 \mathrm{nsec}$ steps with an accuracy of $10 \mathrm{ps}$ in a preset full $10 \mathrm{~ns}$ range. Thus the whole time span between subsequent X-ray pulses can be addressed.

A computer controlled batch routine allows to setup time scans for an automated recording of magnetization dynamic images. It also allows users to apply external 
magnetic static fields, e.g. to saturate the magnetization for normalization purposes or to set a bias field for time resolved magnetization reversal studies.

To determine an absolute time scale, where time zero is defined to be the arrival time of the X-rays onto the sample, an electronic signal of an avalanche photodiode (APD), located close to the sample and triggered by the arriving X-ray pulse is used. The APD signal is simultaneously the pulse monitor signal observed with the fast oscilloscope. The samples in this study consisted of $45 \mathrm{~nm}$ thick rectangular $2 \times 4 \mu \mathrm{m}^{2} \mathrm{Fe}_{20} \mathrm{Ni}_{80}$ patterns aligned on 5nm titanium (Ti)/100nm Au waveguides on top of 80nm silicon nitride $\left(\mathrm{Si}_{3} \mathrm{~N}_{4}\right)$ membranes (Fig. 4). Waveguides were first defined on a standard (100) silicon wafer which had $80 \mathrm{~nm}$ of $\mathrm{Si}_{3} \mathrm{~N}_{4}$ grown on the surface. The waveguides were patterned with UV lithography and a film of $5 \mathrm{~nm} \mathrm{Ti} / 100 \mathrm{~nm} \mathrm{Au}$ was evaporated onto the wafer and followed by liftoff. Membrane patterns were next defined under the waveguides by patterning and etching the backside of the wafer with potassium hydroxide. The sample patterns were aligned to the waveguide structures and defined in poly(methyl methacrylate) with e-beam lithography. Evaporation was used to deposit $45 \mathrm{~nm} \mathrm{Fe}{ }_{20} \mathrm{Ni}_{80}$ and liftoff was done with dichloromethane. Finally, the Si frames with the membranes samples were glued into circuit board holders and the waveguides on the Si frame were wire bonded from pad structures to the circuitry on the board. A standard SMA connector was mounted onto the board to connect standard RF cables to the pulser output.

\section{Results}

The schematic ferromagnetic ground state configuration of the magnetic domain pattern in a $\mathrm{Fe}_{20} \mathrm{Ni}_{80}$ sample with dimensions $2 \mu \mathrm{m}$ x $4 \mu \mathrm{m} \times 45 \mathrm{~nm}$ is shown in Fig. 5a. The magnetization is in plane and the magnetic domain pattern forms a seven domain Landau 
structure consisting of two four domain closure patterns that generally occur in square elements. The spin dynamics in square elements and disks with a high degree of symmetry have been studied recently both experimentally [18- 19] and theoretically [20] To avoid an excess of exchange energy the magnetization curls out of plane at the intersection of the diagonal domain walls in this structure giving rise to two vortex structures in this element.

The time resolved studies with this element were carried out using $854 \mathrm{eV}(1.45 \mathrm{~nm})$ radiation; which corresponds to the nickel $\mathrm{L}_{3}$ edge. To image the in-plane component of the magnetization the sample was placed with a $30^{\circ}$ tilt with respect to the axis of the $\mathrm{X}$ ray beam.

The equation of motion to describe magnetization dynamics is the Landau-LifshitzGilbert equation

$$
\frac{d \vec{M}}{d t}=-\gamma\left[\vec{M} \times H_{e f f}\right]+\frac{\alpha}{|M|}\left[\vec{M} \times \frac{d \vec{M}}{d t}\right] .
$$

where the first term accounts for the precessional motion of the magnetization in an effective local magnetic field $\left(\mathrm{H}_{\mathrm{eff}}\right)$ and $\gamma$ is the gyromagnetic ratio. The second term resembles the damping of the magnetization and the relaxation into the direction of the resulting effective magnetic field. $\alpha$ is a phenomenological parameter describing this relaxation, which depends both on the anisotropy, the geometry and the morphology of the specimen A pronounced example of the magnetic domain pattern at a delay of about $2 \mathrm{nsec}$ after the excitation pulse is shown in Fig. 5b. As indicated by the arrows several distinct features can be observed. The domain wall has bulged outwards as can be seen by the deformed shape of the center domain. Furthermore the high spatial resolution of soft X-ray 
microscopy shows the dislocation of the two vortex structures from their static position, which indicates the gyrotropic vortex motion. The dark area in the center domain connecting two of the closure domains indicates that the two vortices are interacting with each other.

Micromagnetic simulations which are based on the LLG equations support these experimental findings [21]

\section{Conclusion}

Magnetic soft X-ray microscopy is a powerful novel analytical tool to image nanomagnetism with both high spatial resolution and time resolution. XMCD used as magnetic contrast provides a huge contrast mechanism with inherent element sensitivity. This is of advantage e.g. in multicomponent layered structures to achieve layer resolution.

The limited photon flux at current X-ray sources requires a stroboscopic pump-probe scheme and therefore a perfect repeatability of the dynamic process investigated. The time resolution is set by the X-ray source, which is currently at 70ps. Upcoming fsec sources with sufficient photon flux per bunch will allow for single shot imaging of spin dynamics with fsec time resolution.

The spatial resolution is determined by the X-ray optics. $15 \mathrm{~nm}$ have been achieved and the next generation of X-ray optics will lead into the $10 \mathrm{~nm}$ regime.

Thus time resolved soft X-ray microscopy, currently a powerful probe of magnetism, will be a unique tool in the future to record real space images of magnetism down to fundamental length and time scales. 


\section{Acknowledgments}

We thank the staff of CXRO and the ALS, particularly M. -Y. Im, R. Oort, B. Gunion, S. B. Rekawa, and P. Denham for their help in running XM-1. We would also like to thank D. Attwood for his continuous support of our research.

This project is supported by the Director, Office of Science, Office of Basic Energy

Sciences, of the U.S. Department of Energy under Contract No. DE-AC03-76SF00098.

\section{References}

1. S. Bader, Rev. Mod. Phys. 78 (2006) 1

2. G. Srajer et al, J. Magn Magn Mater. 307 (2006) 1

3. Grünberg, P. et al., Phys. Rev. Lett. 57 (1986) 2442

4. Baibich M. et al., Phys.Rev.Lett., 61 (1988) 2472

5. J. C. Slonczewski, J. Magn. Magn. Mater. 159 (1996), L1

6. L. Berger, Phys. Rev. B 54 (1996) 9353

7. S. Mangin et al., Nature Mat 5 (2006) 210

8. M. Hayashi, L. Thomas, C. Rettner, R. Moriya, Y. B. Bazaliy, and S.S.P. Parkin, Phys Rev Lett. 98 (2007) 037204

9. G. Meier, M. Bolte, R. Eiselt, B. Krüger, D.-H. Kim., P. Fischer, Phys Rev Lett. 98 (2007) 187202

10. S. S. P. Parkin, U.S. Patent No. 6834005 (2004)

11. P. Fischer, D.H. Kim, W. Chao, J.A. Liddle, E.H. Anderson, D.T. Attwood, 
Materials Today 9(1-2) (2006)p. 26

12. W. Chao, B.H. Harteneck, J.A. Liddle, E.H. Anderson and D.T. Attwood, Nature 435 (2005), p. 1210

13. D.-H. Kim, P. Fischer, W. Chao, E. Anderson, M.-Y. Im, S.-C. Shin, and S.-B. Choe, J. Appl. Phys. 99 (2006), p. 08H303

14. Chen, C.T. et al., Phys. Rev. B 42 (1990) 7262

15. S.-B. Choe, Y. Acremann, A. Scholl, A. Bauer, A. Doran, J. Stohr, and H.A. Padmore, Science 304, (2004), p 420.

16. J. Raabe et al., Phys. Rev. Lett. 94 (2005) 217204

17. S. Kasai, Y. Nakatani, K. Kobayashi, H. Kohno, T. Ono, Phys Rev. Lett, 97 (2006) 107204

18. K.S. Buchanan, P.E. Roy, M. Grimsditch, F.Y. Fradini, K.Y. Guslienko, S.D. Bader and V. Novosad, Nature Physics 1, (2005) p. 172

19. K.Yu. Guslienko, X. F. Han, D. J. Keavney, R. Divan, S. D. Bader, Phys Rev. Lett, 96 (2006) 067205

20. S Choi, K-S. Lee, K. Yu. Guslienko, S.-K. Kim, Phys Rev Lett, 98 (2007) 087205

21. D.H. Kim, et al, (2007) in preparation 
Figure 1. Schematic setup of the full-field soft X-ray microscopy beamline XM-1 at the Advanced Light Source in Berkeley, CA, USA.

Figure 2. Pump and probe scheme used to image spin dynamics with soft X-ray microscopy. The probing X-ray pulse has a width of 70ps and a $3 \mathrm{MHz}$ frequency. By shifting the time difference between the arrival of the pump and probe, images can be taken of the magnetization as it evolves in time. A typical excitation pulse (pump) is shown.

Figure 3. Signal flow diagram for the pump and probe setup at XM-1.

Figure 4. Diagram of a typical sample arrangement on a waveguide.

Figure 5. Schematic domain configuration in the $2 \times 4 \mu \mathrm{m}^{2}$ permalloy element. The external field direction to saturate the sample is along the long axis, while the exciting magnetic field pulse is along the short axis of the rectangular element. (a) Schematic ground state configuration of the rectangular patterned $\mathrm{Fe}_{20} \mathrm{Ni}_{80}$ element. The arrows indicate the directions of the in-plane magnetization. The tilt axis is vertical, therefore the three center domain with the arrows pointing up/down do not show magnetic contrast in the X-ray image. (b) Representative time-resolved X-ray image obtained of the $2 \times 4 \mu \mathrm{m}^{2}$ rectangular element. Domain configuration $2 \mathrm{~ns}$ after the pulse. The arrows indicate the 
three different features observed: domain wall bulging, gyrotropic vortex motion and vortex-vortex interaction. 


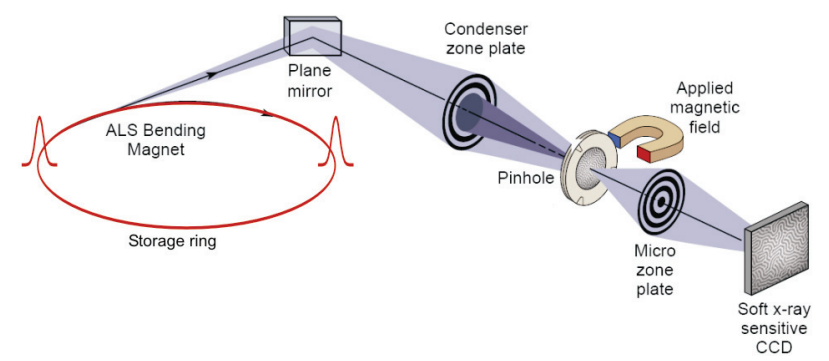

Figure 1 


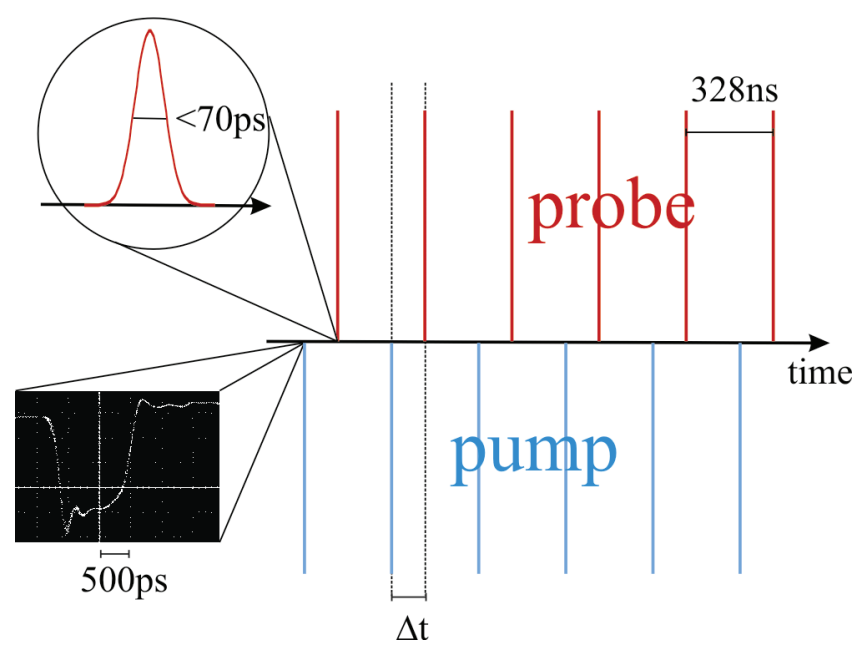

Figure 2 


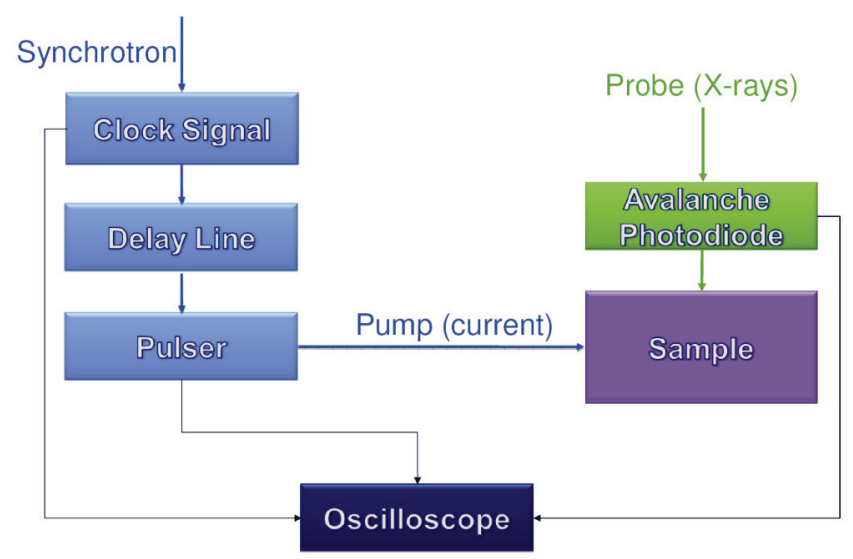

Figure 3 


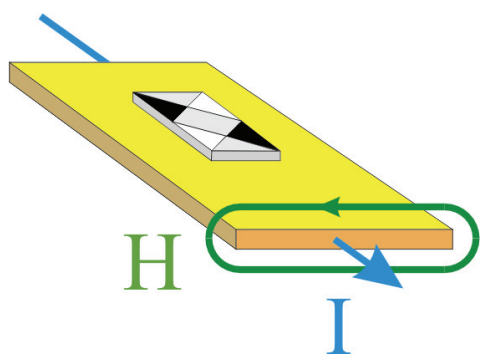

Figure 4 

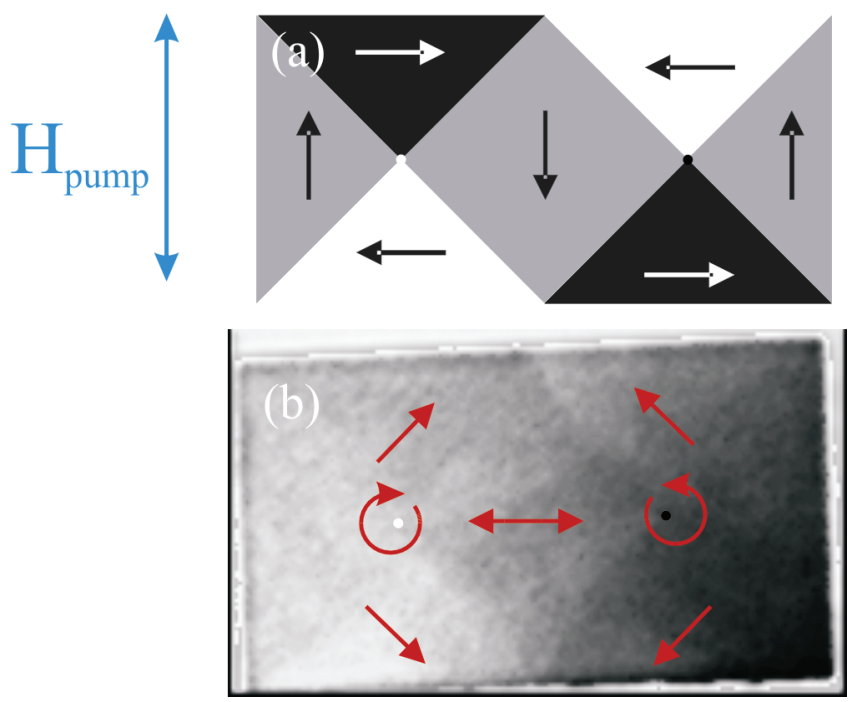

Figure 5 